\title{
Successful treatment of advanced malignant fibrous histiocytoma of the right forearm with apatinib: a case report
}

This article was published in the following Dove Press journal:

OncoTargets and Therapy

5 February 2016

Number of times this article has been viewed

\author{
Guanghui Ji' \\ Liu Hong ${ }^{2}$ \\ Ping Yang ${ }^{3}$ \\ 'Department of Traditional Chinese \\ Medicine, ${ }^{2}$ Department of Pathology, \\ ${ }^{3}$ Department of Oncology, Navy \\ General Hospital, Beijing, People's \\ Republic of China
}

\begin{abstract}
Malignant fibrous histiocytoma (MFH) is the most common soft-tissue sarcoma in late adult life. Unfortunately, advanced MFH has a poor prognosis due to a lack of effective drugs. We present here a case of advanced MFH with partial response to apatinib, a new potent oral small-molecule tyrosine kinase inhibitor targeting the intracellular domain of vascular endothelial growth factor receptor 2 (VEGFR-2). To the best of our knowledge, this is the first case report using apatinib for MFH. Quantitative polymerase chain reaction analysis revealed high expression of VEGFR-2 mRNA, suggesting that apatinib leads to clinical response by inhibiting VEGFR-2 tyrosine kinase activity and the crucial role of VEGFR-2 for MFH. Apatinib could be a new option for the treatment of MFH. Further studies are needed to optimize the treatment.
\end{abstract}

Keywords: malignant fibrous histiocytoma, apatinib, chemotherapy, angiogenesis inhibitor, targeted therapy

\section{Introduction}

Malignant fibrous histiocytoma (MFH), originated from mesenchymal cells, is the most common soft-tissue sarcoma (STS) in late adult life. It frequently occurs in deep soft tissues of the extremities $(70 \%){ }^{1}$

Standard treatment guidelines for MFH do not exist currently. Surgical management remains the main treatment. Unfortunately, approximately $40 \%$ of patients will develop local recurrences or distant metastases. ${ }^{2}$ The 5 -year survival rate is $10 \%-30 \%$ if MFH is only treated locally. ${ }^{3}$ Radiotherapy and chemotherapy in adjuvant settings has only limited effectiveness. ${ }^{4}$ For advanced MFH, the prognosis of MFH remains very poor, even with combined, multimodal therapy. There is an urgent need for novel effective agents.

Apatinib (Hengrui Pharmaceutical Co., Ltd, Shanghai, People's Republic of China) is a novel oral small-molecule tyrosine kinase inhibitor (TKI) targeting the intracellular domain of vascular endothelial growth factor receptor-2 (VEGFR-2). It has shown a survival benefit in gastric cancer in a Phase III trial and non-small-cell lung cancer in a Phase II trial and is currently being studied in multiple tumor types. Because of low side effects and improved outcomes, apatinib has demonstrated a substantial potential to be a new therapeutic option in a variety of tumor types. ${ }^{5}$

We here report a case of $\mathrm{MFH}$ on the right forearm with partial response (PR) to apatinib. To the best of our knowledge, this is the first case of MFH that responded to apatinib.
Department of Oncology, Navy General Hospital, 6 Fucheng Road, Haidian

District, Beijing 100048,

People's Republic of China

Tel +86 I86 003। 097|

Email jgh.2007@।63.com 


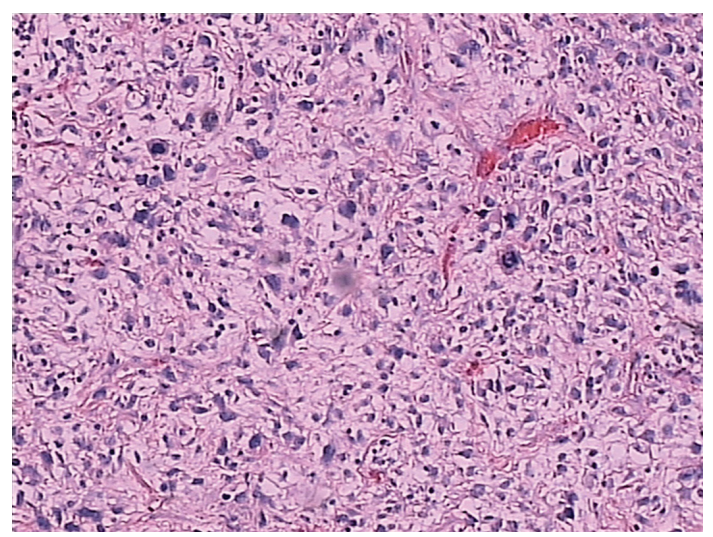

Figure I Hematoxylin and eosin staining of a tumor section $(\times 200)$.

\section{Case report}

In May 2013, a 78-year-old male came to our hospital with an irregular soft-tissue mass on the right forearm over 1 year, with an area of approximately $6.0 \mathrm{~cm} \times 5.0 \mathrm{~cm}$.

The biopsy from the lesions was taken, and histopathologic examination revealed MFH. The presence of distal metastases was ruled out by chest and abdominal computed tomography (CT) at that time. However, the patients did not receive surgical excision until December 23, 2013, for personal reasons. The mass was found between skin and fascia at operation, with a volume of $5 \mathrm{~cm} \times 4 \mathrm{~cm} \times 3 \mathrm{~cm}$. However, complete resection was not achieved because of nerve infiltration. The postoperative pathology revealed positive surgical margins on the edge of the skin and the basal layer of epithelium. Pathology diagnosis confirmed MFH (mostly myxofibrosarcoma, some pleomorphic forms; Figure 1) with immunophenotype: Vimentim(3+), AAT(2+), AE1/AE3(-), CD34(3+), SMA(-), Bcl2( \pm ), S100(-), Desmin(-), LCA(-), $\operatorname{ALK}(-), \operatorname{CD} 30(-), \beta$-catenin(+), CD68(+).

The patient was then referred to a radiation oncologist to receive a total of 60 Gy of intensity modulation radiated therapy.

One year after the operation, chest CT (December 10, 2014) revealed multiple lung nodules, considering the possibility of metastases. Whole-body positron emission tomography (PET)/CT further confirmed multiple lung metastases with increased glucose metabolism without local recurrence and metastases in other parts of the body. The patient refused to receive cytotoxic chemotherapy, instead opting for some traditional Chinese medicine because of old age and poor performance status (PS). One month later (November 10, 2014), chest CT revealed increased metastases.

Quantitative polymerase chain reaction of VEGFR-2 mRNA and gene sequencing of EGFR and KRAS were then performed, and the results revealed high expression of VEGFR-2 mRNA, wild-type EGFR, and wild-type KRAS.

By December 19, 2014, the patient had symptoms of intermittent expectoration and hemoptysis. Chest $\mathrm{CT}$ revealed an increased metastasis in the upper lobe of right lung and a significantly increased mass of approximately $9.3 \mathrm{~cm}$ in diameter at the lower right parahilar (Figure 2A and B).

After the patient provided written, informed consent, apatinib was then administered with a dose of $500 \mathrm{mg} / \mathrm{d}$ on December 28, 2014. Following 2 months of targeted therapy, symptoms of expectoration reduced significantly and no
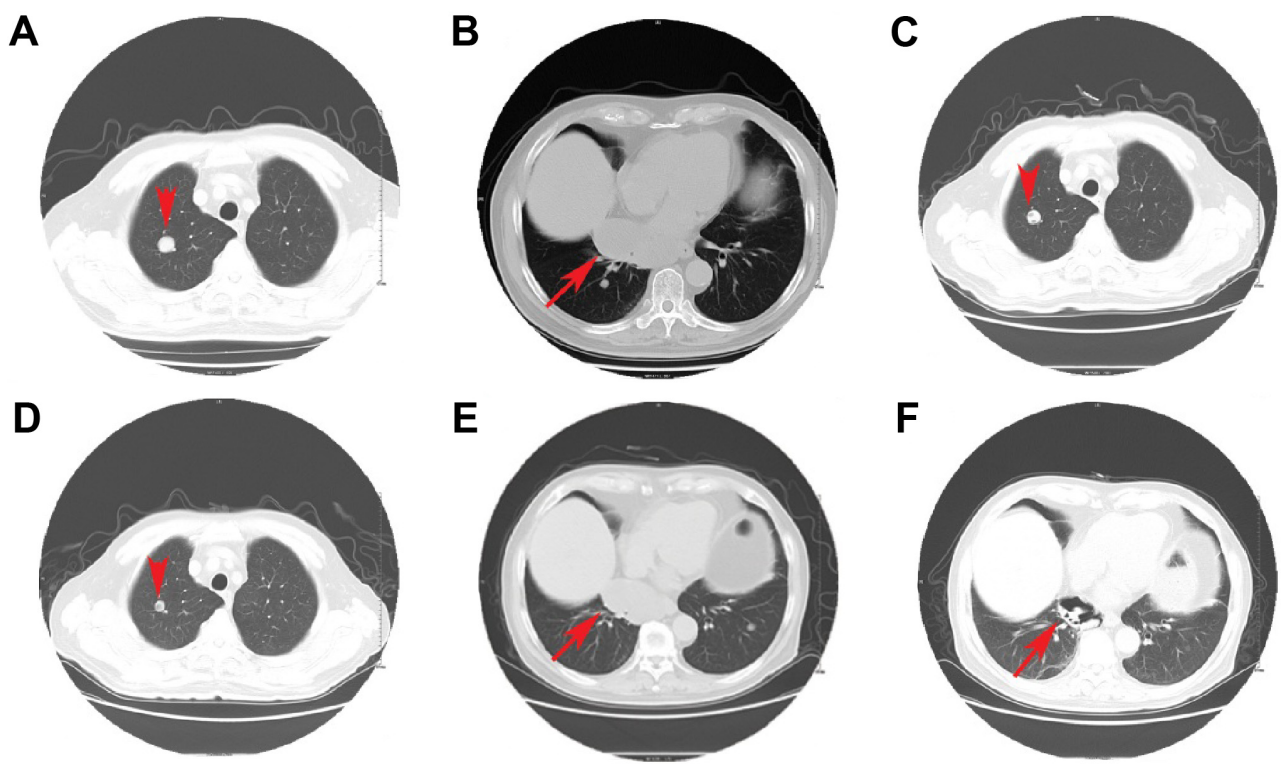

Figure 2 Chest CT images showing metastases before (A and $\mathbf{B})$ and after treatment with apatinib (C, D and E, F, respectively).

Note: The arrows and arrowheads indicate the pulmonary metastases.

Abbreviation: CT, computed tomography. 
A

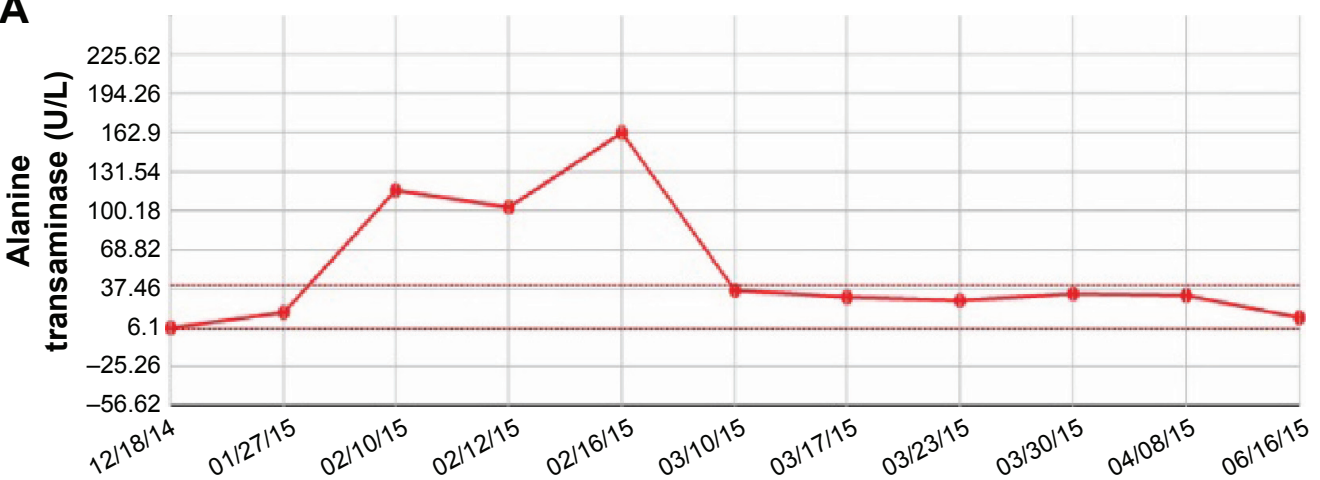

B

Dates

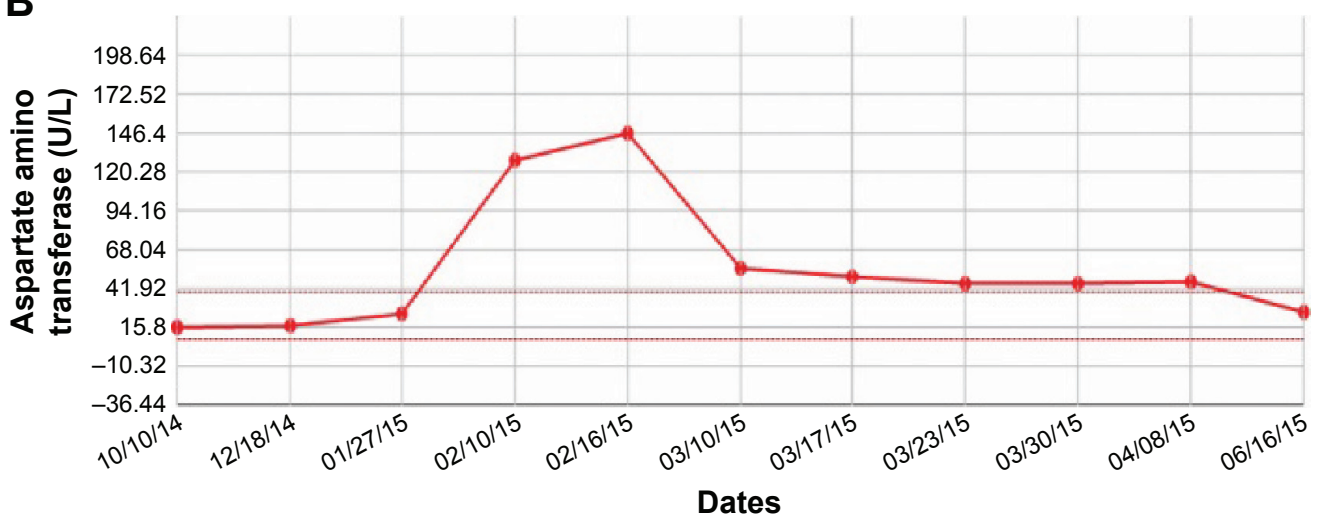

Figure 3 Main toxicities during apatinib treatment.

Notes: (A) Elevated alanine transaminase. (B) Elevated aspartate amino transferase.

hemoptysis appeared. Lung metastases were significantly reduced in size with some porosis, which was considered to be a PR (Figure 2C, D and E, F, respectively).

The toxicities the patient experienced were mainly nonhematological toxicities, including skin rash, short-lived elevated alanine transaminase (Figure 3A), and aspartate amino transferase (Figure 3B; grade 2; according to Common Terminology Criteria for Adverse Events v.4.03). No severe toxicities (grades 3-4) and other treatment-related adverse events were reported and detected.

The patient continued to use apatinib as maintenance therapy. A progression-free survival time of more than 6 months has been achieved and the patient is now still undergoing the apatinib treatment without major toxic effects.

\section{Discussion}

MFH is the commonest STS. It usually occurs in late adulthood with a slight predominance in males. ${ }^{6}$

There are no standard treatment guidelines for MFH. Successful en-bloc resection of the tumor is the principal treatment and is the most important prognostic factor. ${ }^{7}$ The role of adjuvant chemotherapy and radiation is limited and controversial. Even with en bloc resection (better results than debulking surgery in terms of survival),${ }^{8}$ many patients suffered from metastases to lungs, bone, liver, and skin. ${ }^{9}$

In the advanced stage, the standard treatment is chemotherapy. Regimens are primarily based on doxorubicin and ifosfamide as first-line treatment, either alone or in combination. Approximately $30 \%$ of patients treated with doxorubicin-based therapy or ifosfamide-based therapy achieve an objective response. ${ }^{10}$ The combination of the two drugs could offer a response rate of approximately $30 \%-59 \%$ in patients with STS. ${ }^{11}$ In a single institution study of 33 patients with STS, approximately $60 \%$ of patients treated with dose-intense doxorubicin and ifosfamide achieved an objective response, but $31 \%-56 \%$ of patients suffered from febrile neutropenia. ${ }^{12}$ However, doxorubicin in combination with ifosfamide did not improve 1-year survival. ${ }^{13}$

Other chemotherapy options in STS include either gemcitabine monotherapy or docetaxel and gemcitabine combination therapy. ${ }^{14,15}$ The activity of docetaxel monotherapy is quite limited. In a randomized Phase II study of docetaxel versus doxorubicin in first- and second-line chemotherapy for locally advanced or metastatic STS in Europe, no responses $(0 \%)$ were seen in the docetaxel therapy arm, compared to the response rate of $30 \%$ in doxorubicin therapy arm. ${ }^{14}$ Response rates of gemcitabine 
monotherapy in patients with advanced STS were reported to be $3 \%-20.5 \%$, with median time to progression $1.5-13$ months and median overall survival 6-15 months. ${ }^{16-18}$ Although with limited efficacy, docetaxel may have a synergistic action with gemcitabine when given before gemcitabine. In an open-label Phase II clinical trial with metastatic STS, the overall objective response rates were $16 \%$ (gemcitabine-docetaxel) and $8 \%$ (gemcitabine), with a response rate of $25 \%$ with gemcitabine and $36 \%$ with the combination for $\mathrm{MFH}$, indicating that $\mathrm{MFH}$ is sensitive to both gemcitabine and the gemcitabine plus docetaxel combination. ${ }^{19}$ However, combination of gemcitabine with docetaxel is potentially associated with significant toxicity. More than $50 \%$ of patients receiving the combination of gemcitabine and docetaxel discontinued treatment within 6 months, despite dose reductions. ${ }^{20}$

Considering that the prevalence of $\mathrm{MFH}$ is highest in late adulthood, and that the toxicities of combined chemotherapy often leads to treatment cessation, MFH requires novel, effective, and safer treatment options, especially for the aged.

The advances in understanding the molecular mechanisms of malignancies have led to the development of targeted treatment options. Targeted TKIs represent a major treatment of choice. Several potential TKIs have been reported to be effective in the treatment of MFH, including sunitinib, sorafenib, and pazopanib. ${ }^{21-25}$ Moreover, preclinical data evidenced that imatinib could reduce tumor growth of MFH that express PDGFRs and c-Kit. ${ }^{26}$ However, no case has been reported for apatinib in the treatment of MFH.

Apatinib is a novel oral small-molecule protein tyrosine kinase (RTK) inhibitor that selectively targets VEGFR-2, the primary VEGF family receptor driving angiogenesis. ${ }^{27}$ VEGFR-2 binds all VEGF-A isoforms, VEGF-C and VEGF-D. The binding of VEGF-A to VEGFR-2 could induce a cascade of different signaling pathways such as PLC- $\gamma$-Raf kinaseMEK-MAP kinase and PI3K-AKT pathways, eventually causing cellular proliferation and endothelial-cell survival. A number of clinical trials have confirmed the efficacy of blockage of VEGFR-2 to be a promising strategy for inhibiting angiogenesis. ${ }^{28}$ Apatinib has shown a survival benefit in gastric cancer in a Phase III trial and in a non-small-cell lung cancer in a Phase II trial in China, with a manageable toxicity profile. ${ }^{5}$

In the Phase I study of apatinib, dose escalation was conducted in patients aged between 18 and 70 years. Hypertension and hand-foot syndrome were the two doselimiting toxicities noted at dose level of 1,000 mg. Maximum tolerated dose was determined to be $850 \mathrm{mg}$ once daily. The recommended dose of $750 \mathrm{mg}$ once daily was well tolerated.
The most frequently observed drug-related adverse events were hypertension (69.5\%), proteinuria (47.8\%), and handfoot syndrome $(45.6 \%){ }^{29}$

In this case, the patient with MFH did not receive en bloc resection because of nerve infiltration. Although radiotherapy was performed, multiple lung metastases developed 1 year after the surgery. Because of old age and poor PS, the patient refused to accept any toxic chemotherapy, and apatinib monotherapy was then used to control the disease successfully with a PR.

Gene expression analysis revealed high expression of VEGFR-2 mRNA, suggesting that apatinib led to clinical response by inhibiting VEGFR-2 tyrosine kinase activity and the crucial role of VEGFR-2 for MFH. The administration of apatinib resulted in well-tolerated toxic profiles.

\section{Conclusion}

In conclusion, apatinib may provide an additional option for the treatment of MFH especially for the aged and patient with poor PS. Nevertheless, further large-scale prospective studies are required to improve the treatment of angiosarcoma.

\section{Acknowledgments}

This case report was approved by the Medical Ethics Committee of the Navy General Hospital. Written informed consent was obtained from the patient for publication of this case report and accompanying images. The authors thank the patient for his participation and his agreement to publication of the report.

\section{Author contributions}

All authors contributed toward data analysis, drafting and critically revising the paper, gave final approval of the version to be published, and agree to be accountable for all aspects of the work.

\section{Disclosure}

The authors report no conflicts of interest in this work.

\section{References}

1. Abou-Jaoude M, El Ali M. Malignant fibrous histiocytoma: a case report and literature review. Int Surg. 2009;94(3):196-200.

2. Weitz J, Antonescu CR, Brennan MF. Localized extremity soft tissue sarcoma: improved knowledge with unchanged survival over time. J Clin Oncol. 2003;21(14):2719-2725.

3. Bacci G, Avella M, Picci P, et al. The effectiveness of chemotherapy in localized malignant fibrous histiocytoma (MFH) of bone: the Rizzoli Institute experience with 66 patients treated with surgery alone or surgery + adjuvant or neoadjuvant chemotherapy. Chemioterapia. 1988; 7(6):406-413. 
4. Efe T, Heyse TJ, Schofer MD, Fuchs-Winkelmann S, Rexin P, Schmitt J. Malignant fibrous histiocytoma of the distal femur after an arthroscopic anterior cruciate ligament reconstruction: a case report and a review of the literature. BMC Cancer. 2010;10:264.

5. Scott AJ, Messersmith WA, Jimeno A. Apatinib: a promising oral antiangiogenic agent in the treatment of multiple solid tumors. Drugs Today (Barc). 2015;51(4):223-229.

6. Pobirci DD, Bogdan F, Pobirci O, Petcu CA, Rosca E. Study of malignant fibrous histiocytoma: clinical, statistic and histopatological interrelation. Rom J Morphol Embryol. 2011;52(1 Suppl):385-388.

7. Teng H, Xinghai Y, Wei H, Huang Q, Xiao J, Zhang C. Malignant fibrous histiocytoma of the spine: a series of 13 clinical case reports and review of 17 published cases. Spine (Phila Pa 1976). 2011;36(22): E1453-E1462.

8. Kawahara N, Tomita K, Murakami H, Demura S. Total en bloc spondylectomy for spinal tumors: surgical techniques and related basic background. Orthop Clin North Am. 2009;40(1):47-63, vi.

9. Sereno M, Merino M, Aguayo C, et al. A major response to trabectedin in metastatic malignant fibrous histiocytoma of the vertebra: a case report and review of the literature. Tumori. 2013;99(2):e43-e48.

10. Kanjeekal S, Chambers A, Fung MF, Verma S. Systemic therapy for advanced uterine sarcoma: a systematic review of the literature. Gynecol Oncol. 2005;97(2):624-637.

11. Wei Z, Li L, Xu XY, et al. A case of recurrent malignant fibrous histiocytoma with marked response to combined chemotherapy with gemcitabine and carboplatin. Arch Med Sci. 2014;10(5):1057-1060.

12. Patel SR, Vadhan-Raj S, Burgess MA, et al. Results of two consecutive trials of dose-intensive chemotherapy with doxorubicin and ifosfamide in patients with sarcomas. Am J Clin Oncol. 1998;21(3):317-321.

13. Verma S, Younus J, Stys-Norman D, Haynes AE, Blackstein M. Metaanalysis of ifosfamide-based combination chemotherapy in advanced soft tissue sarcoma. Cancer Treat Rev. 2008;34(4):339-347.

14. Verweij J, Lee SM, Ruka W, et al. Randomized phase II study of docetaxel versus doxorubicin in first- and second-line chemotherapy for locally advanced or metastatic soft tissue sarcomas in adults: a study of the european organization for research and treatment of cancer soft tissue and bone sarcoma group. J Clin Oncol. 2000;18(10):2081-2086.

15. Seddon B, Scurr M, Jones RL, et al. A phase II trial to assess the activity of gemcitabine and docetaxel as first line chemotherapy treatment in patients with unresectable leiomyosarcoma. Clin Sarcoma Res. 2015;5:13

16. Look KY, Sandler A, Blessing JA, Lucci JA 3rd, Rose PG. Phase II trial of gemcitabine as second-line chemotherapy of uterine leiomyosarcoma: a Gynecologic Oncology Group (GOG) Study. Gynecol Oncol. 2004; 92(2):644-647.
17. Hartmann JT, Oechsle K, Huober J, et al. An open label, non-comparative phase II study of gemcitabine as salvage treatment for patients with pretreated adult type soft tissue sarcoma. Invest New Drugs. 2006; 24(3):249-253.

18. Okuno S, Ryan LM, Edmonson JH, Priebat DA, Blum RH. Phase II trial of gemcitabine in patients with advanced sarcomas (E1797): a trial of the Eastern Cooperative Oncology Group. Cancer. 2003;97(8): 1969-1973.

19. Maki RG, Wathen JK, Patel SR, et al. Randomized phase II study of gemcitabine and docetaxel compared with gemcitabine alone in patients with metastatic soft tissue sarcomas: results of sarcoma alliance for research through collaboration study 002 [corrected]. J Clin Oncol. 2007;25(19):2755-2763.

20. Maki RG. Gemcitabine and docetaxel in metastatic sarcoma: past, present, and future. Oncologist. 2007;12(8):999-1006.

21. Caspersen CS, Sosunov A, Utkina-Sosunova I, Ratner VI, Starkov AA, Ten VS. An isolation method for assessment of brain mitochondria function in neonatal mice with hypoxic-ischemic brain injury. Dev Neurosci. 2008;30(5):319-324.

22. Mauri D, Panou C, Valachis A, Kamposioras K, Tsali L. Tyrosine kinase inhibitors in treatment of fibrous histiocytoma. Exp Oncol. 2009;31(1):60-61

23. Maki RG, D’Adamo DR, Keohan ML, et al. Phase II study of sorafenib in patients with metastatic or recurrent sarcomas. J Clin Oncol. 2009;27(19):3133-3140. doi:3110.1200/JCO.2008.3120.4495.

24. Heudel P, Cassier P, Derbel O, et al. Pazopanib for the treatment of soft-tissue sarcoma. Clin Pharmacol. 2012;4:65-70.

25. Rajendra R, Jones RL, Pollack SM. Targeted treatment for advanced soft tissue sarcoma: profile of pazopanib. Onco Targets Ther. 2013;6: 217-222.

26. Irsan I, Akisue T, Hara H, et al. Imatinib mesylate inhibits tumorigenicity of malignant fibrous histiocytoma cells in vivo. Anticancer Res. 2007; 27(1A):423-429.

27. Tian S, Quan H, Xie C, et al. YN968D1 is a novel and selective inhibitor of vascular endothelial growth factor receptor-2 tyrosine kinase with potent activity in vitro and in vivo. Cancer Sci. 2011;102(7): 1374-1380.

28. Fontanella C, Ongaro E, Bolzonello S, Guardascione M, Fasola G, Aprile G. Clinical advances in the development of novel VEGFR2 inhibitors. Ann Transl Med. 2015;2(12):123.

29. Li J, Zhao X, Chen L, et al. Safety and pharmacokinetics of novel selective vascular endothelial growth factor receptor-2 inhibitor YN968D1 in patients with advanced malignancies. BMC Cancer. 2010;10:529.
OncoTargets and Therapy

\section{Publish your work in this journal}

OncoTargets and Therapy is an international, peer-reviewed, open access journal focusing on the pathological basis of all cancers, potential targets for therapy and treatment protocols employed to improve the management of cancer patients. The journal also focuses on the impact of management programs and new therapeutic agents and protocols on

\section{Dovepress}

patient perspectives such as quality of life, adherence and satisfaction. The manuscript management system is completely online and includes a very quick and fair peer-review system, which is all easy to use. Visit http://www.dovepress.com/testimonials.php to read real quotes from published authors. 